\title{
Adjustable Multilayer HTS Filters
}

\author{
Kouth Chen, Ming-Jye Chen, Jau-Han Chen, Student Member, IEEE, Hong-Chang Yang, Li-Min Wang, \\ Chao-Yuan Huang, and Bert Wang
}

\begin{abstract}
An adjustable multilayer high-temperature superconductor (HTS) bandpass filter has been fabricated. Taped comb-line filters with multilayer structures were designed. $\mathrm{YBa}_{2} \mathrm{Cu}_{3} \mathrm{O}_{7-\delta}$ (YBCO) thin films deposited on $\mathrm{LaAlO}_{3}$ were used to construct the packaged multilayer HTS filter. The electrical length of the resonators in the filter was smaller than a quarter-wave length. The frequency responses of the filter were measured at liquid nitrogen temperature $77 \mathrm{~K}$. The insertion loss of the packaged filter was determined to be less than $0.5 \mathrm{~dB}$. By mechanically adjusting the multilayer structure, the center frequencies of the filter changed from 1.78 to $1.92 \mathrm{GHz}$, and the variations of the bandwidth from 60 to $150 \mathrm{MHz}$ were also obtained.
\end{abstract}

Index Terms-Adjustable, filter, high-temperature superconductors, multilayer structure.

\section{INTRODUCTION}

$\mathbf{I}$ $\mathrm{N}$ WIRELESS communications, high-temperature superconductor (HTS) filters, fitted in cryocoolers as front-end receivers, have been widely tested [1], [2]. It has been proved that HTS filters have extremely steep rejection skirts at the bandpass edge and a nearly flat response across the passband. With regard to the cryogenic environment for HTS filters, a large device size will cause heavy refrigeration loading. Therefore, developing HTS filters to a minimum size is important for reducing the cooling space and power consumption of the refrigeration system. Hairpin-comb filters [3] and hairpin-resonator filters [4] were previously proposed to minimize the configuration of HTS microstrip filters.

In this paper, we report the design and fabrication of a new adjustable multilayer HTS filter. It is based on a taped-comb-line filter with layered loading capacitors. The electrical length of the resonators can be dramatically decreased when the loading capacitors are greatly increased. The size of the filter is then reduced. By moving the position of the individual layer and changing the loading capacitors, the center frequency of the filter can be adjusted. The bandwidth can also be varied by mechanically adjusting the height of the gap between the filter patterns and the housing tip.

Manuscript received March 15, 2001

K. Chen and B. Wang are with the Advanced Superconducting Technologies Co., Ltd., Taipei 11006, Taiwan.

M.-J. Chen, J.-H. Chen, and H.-C. Yang are with the Department of Physics, National Taiwan University, Taipei 10617, Taiwan.

L.-M. Wang is with the Department of Electrical Engineering, Da-Yeh University, Chang-Hwa 51505, Taiwan.

C.-Y. Huang is with the Center for Condensed Matter Science, National

Taiwan University, Taipei 10617, Taiwan.

Publisher Item Identifier S 1051-8223(01)07087-7.

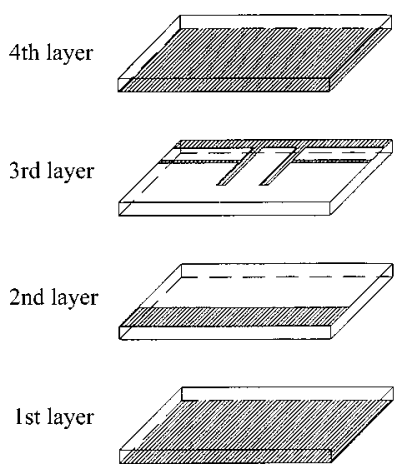

Fig. 1. The schematic of four HTS YBCO films stacked to form a multilayer HTS filter.

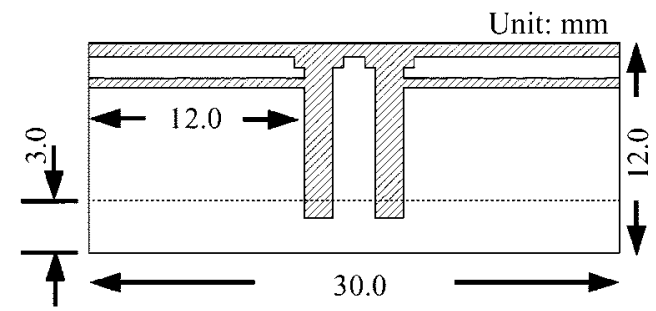

Fig. 2. Detailed filter patterns on the second and third layers. The dashed rectangle was the capacitor ground on the second layer.

\section{EXPERIMENT}

A packaged multilayer HTS filter composed of four $\mathrm{YBa}_{2} \mathrm{Cu}_{3} \mathrm{O}_{7-\delta}$ (YBCO) films in a copper housing was designed. The dimensions of the package inner space were 30 $\times 12 \times 7 \mathrm{~mm}$. Three conductor layers were stacked on the bottom surface and one layer was attached to the top surface of the package space. The schematic representation of the layered structure is shown in Fig. 1. The first conductor is used as the bottom ground, the second one as a capacitor ground, the third one contains resonator patterns, and the fourth one is the top ground. The detailed filter pattern, on the second and third conductor level, is described in Fig. 2. The dashed rectangle was the capacitor ground plane on the second layer. On the third layer, the $12-\mathrm{mm}$ long and $0.6-\mathrm{mm}$ wide of input-output microstrip lines were perpendicularly connected to the two resonator patterns, respectively. The top ends of the resonators were respectively connected to a long rectangular pattern having one end connected to the ground. In the vertical direction, there is an air gap between the third and the fourth conductor levels for frequency response tuning. The filter was simulated by using 2.5-D simulators contained in the software 


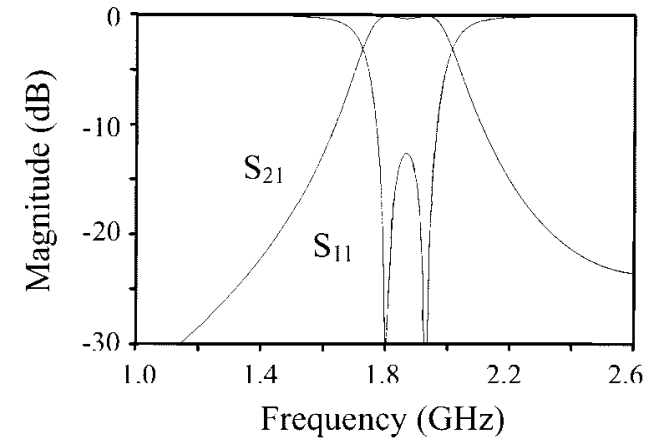

Fig. 3. Simulated frequency responses of a two-stage bandpass filter.

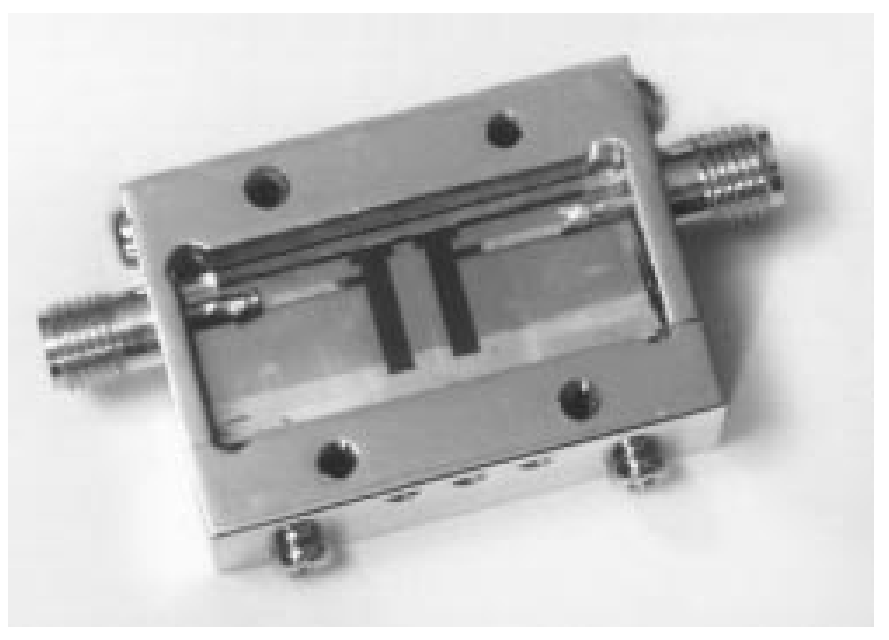

Fig. 4. Prototype of complete packaged adjustable multilayer HTS filters.

LINMIC+/N (by JANSEN Microwave GmbH, Germany). The designed filter was a two-stage Tchebyscheff bandpass with the center of frequency $\left(f_{0}\right)$ at $1.86 \mathrm{GHz}$ and bandwidth (BW) of $120 \mathrm{MHz}$. Fig. 3 shows the modeled frequency responses of the two-pole filter.

Commercial YBCO films deposited on 0.5-mm-thick $\mathrm{LaAlO}_{3}$ substrates $\left(\varepsilon_{r}=24\right)$ were used in this experiment. The specifications of the films are $T_{c}>87 \mathrm{~K}, J_{c} \sim 2 \times 10^{6}$ $\mathrm{A} / \mathrm{cm}^{2}$ at $77 \mathrm{~K}$, and $R_{s} \leq 0.6 \mathrm{~m} \Omega$ at $10 \mathrm{GHz}$ and $77 \mathrm{~K}$ (from THEVA GmbH, Germany). The loss tangent $\left(\tan \delta\right.$ ) of $\mathrm{LaAlO}_{3}$ was reported to be smaller than $10^{-5}$ at $6.3 \mathrm{GHz}$ and $77 \mathrm{~K}$ [5]. The usual photolithographic technique with wet etching method was employed to pattern the YBCO films. A surface profilometer was used to measure a post-etching strip with a designed width of $300 \mu \mathrm{m}$. The width was determined to be $295 \mu \mathrm{m}$ and the thickness was about $0.83 \mu \mathrm{m}$. Four YBCO films were stacked in a copper housing to form a multilayer HTS filter. Gold films with $0.2 \mu \mathrm{m}$ in thickness were deposited on the input-output of the filter patterns as the pattern contact points. Two SMA connectors were attached to the copper housing as the input-output terminals. In-Sn solder was used to connect the SMA pins and the input-output of the filter patterns. Ag-paint with contact resistivity $\rho \sim 1 \times 10^{-4} \Omega \cdot \mathrm{cm}$ was used to short HTS transmission lines to ground. Fig. 4 shows the photo of a complete multilayer HTS filter.

A Wiltron 360B vector network analyzer (VNA) was used to measure the (reverse) reflection coefficient $\left(\mathrm{S}_{22}\right) \mathrm{S}_{11}$ and (re-

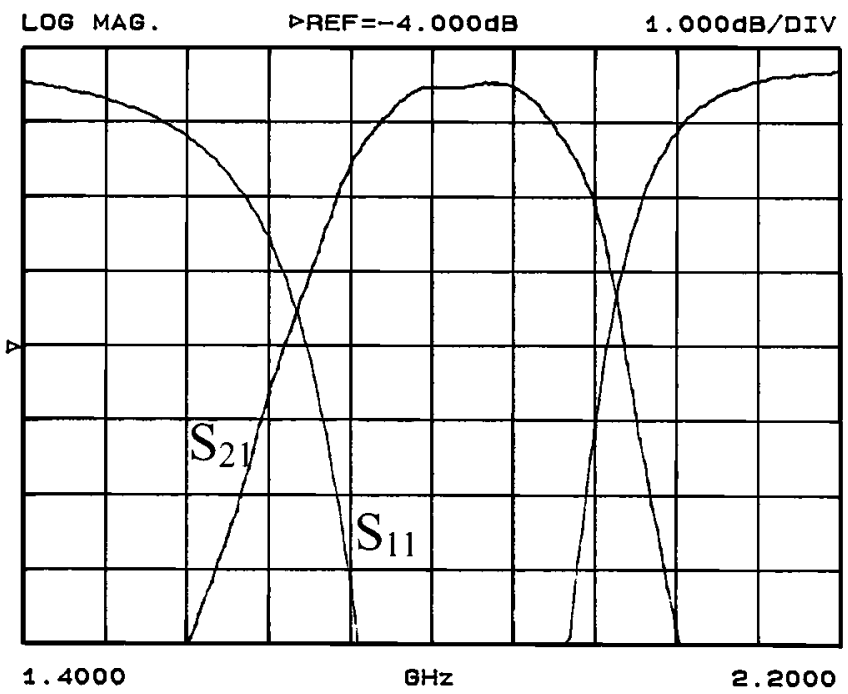

(a)

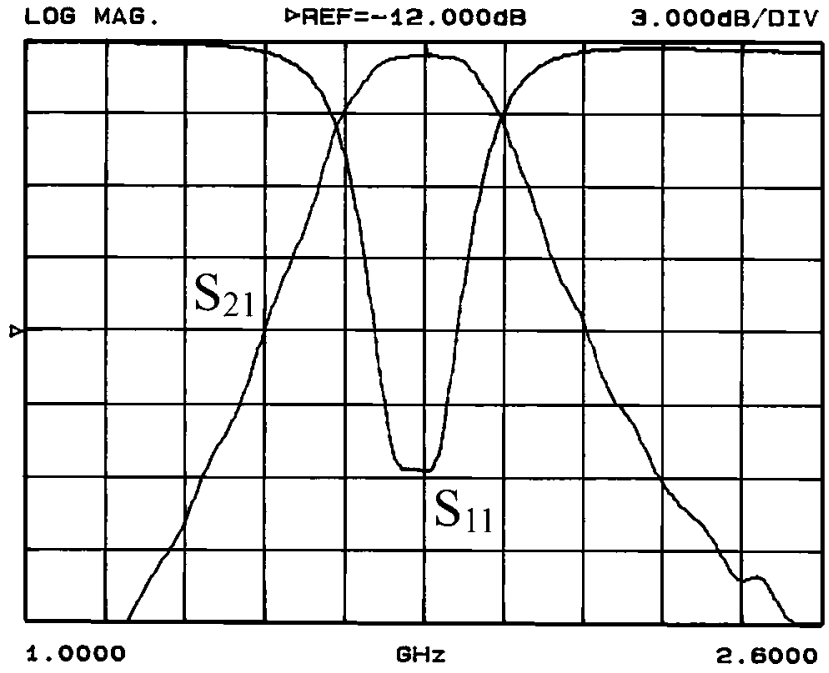

(b)

Fig. 5. (a) Frequency responses of the adjustable multilayer HTS filter, with $f_{0}=1.83 \mathrm{GHz}, \mathrm{BW}=60 \mathrm{MHz}$, and $\mathrm{IL}=0.45 \mathrm{~dB}$. (b) Varied frequency responses of the HTS filter, $f_{0}$ decreased from 1.83 to $1.78 \mathrm{GHz}$ with $\mathrm{BW}=60$ $\mathrm{MHz}$ and $\mathrm{IL}=0.59 \mathrm{~dB}$.

verse) transmission coefficient $\left(\mathrm{S}_{12}\right) \mathrm{S}_{21}$. A pair of cryogenic measurement cables $\left(+150{ }^{\circ} \mathrm{C}\right.$ to $\left.-269^{\circ} \mathrm{C}\right)$ was connected to the input-output ends of VNA with each individual end. The other ends were, respectively, connected to the SMA connectors of the packaged HTS filter. The filter with connected cables was then immersed into liquid nitrogen $(77 \mathrm{~K})$ to do measurements.

\section{RESULTS AND DISCUSSION}

Fig. 5(a) represents a measurement of frequency response of the packaged multilayer HTS filter. The center of frequency was at $1.83 \mathrm{GHz}$, the bandwidth was $60 \mathrm{MHz}$, and the insertion loss (IL) was $0.45 \mathrm{~dB}$ at $1.86 \mathrm{GHz}$. For varying the loading capacitors of the designed comb-line filter, the superconducting patterns on the third layer were moved. Moving the two patterns on layer 2 and layer 3 closer to each other by $0.5 \mathrm{~mm}$, the loading capacitors of the filter were increased, and the center of frequency was then decreased. Fig. 5(b) shows the frequency shifting. The 


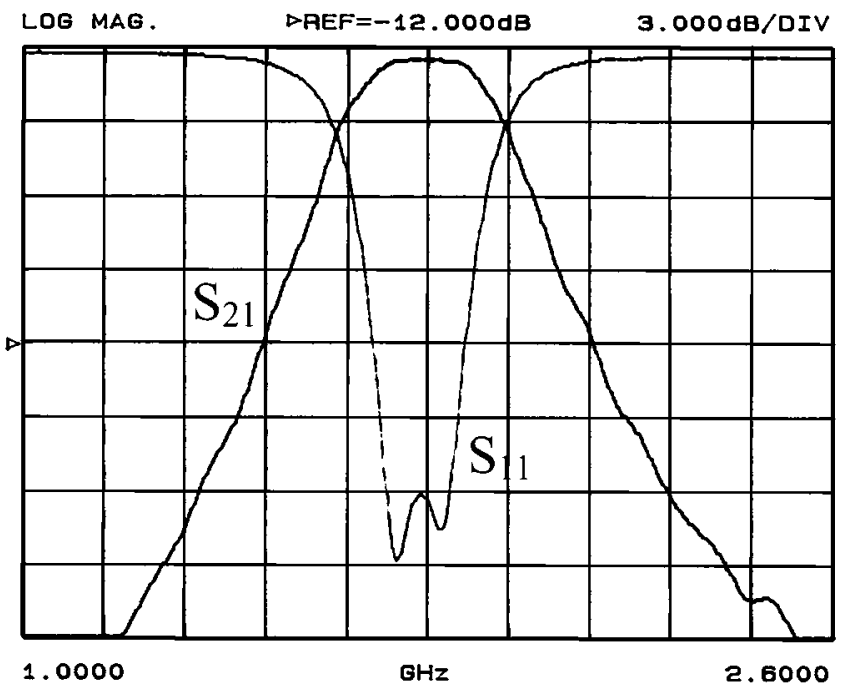

(a)

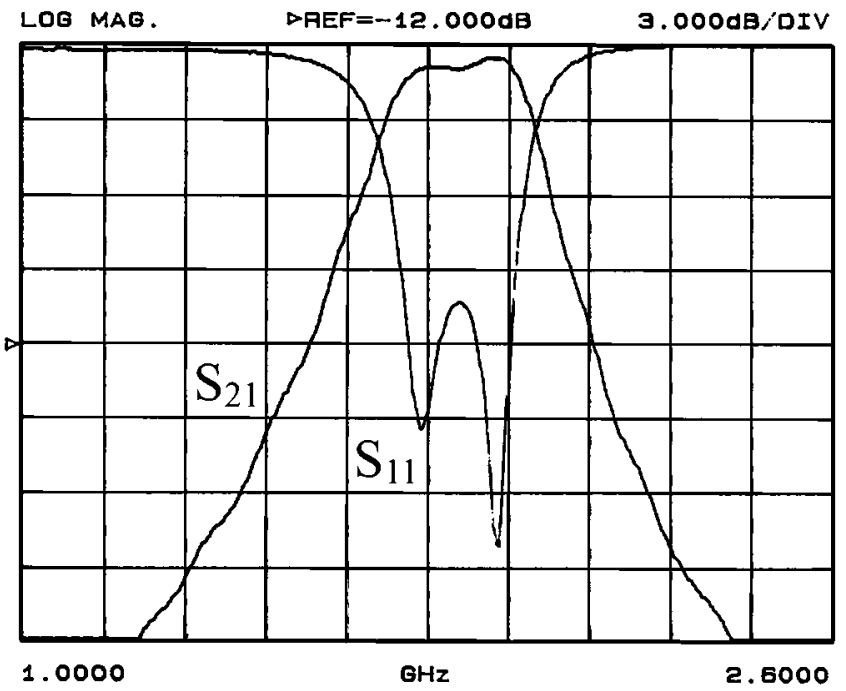

(b)

Fig. 6. (a) Varied frequency response of the HTS filter with VD $=5 \mathrm{~mm}$, showing $\mathrm{BW}=110 \mathrm{MHz}, f_{0}=1.78 \mathrm{GHz}$, and $\mathrm{IL}=0.50 \mathrm{~dB}$. (b) Varied frequency response of the HTS filter with VD $=6 \mathrm{~mm}$, showing BW $=150 \mathrm{MHz}, f_{0}=1.86 \mathrm{GHz}$, and $\mathrm{IL}=0.52 \mathrm{~dB}$.

center frequency of the HTS filter was varied from 1.83 to 1.78 $\mathrm{GHz}$ with BW of $60 \mathrm{MHz}$. The center frequency can also be adjusted toward higher frequency by reducing the loading capacitors. In this experiment, we obtained a center frequency of $1.92 \mathrm{GHz}$ with $\mathrm{IL}=0.56 \mathrm{~dB}$.

The bandwidth of the HTS filter has been adjusted by varying the vertical distance (VD, air gap) of the third and the fourth conducting levels. The bandwidth of the filter changed from 60 to $150 \mathrm{MHz}$. The frequency response shown in Fig. 5(b) corresponds to a filter with $\mathrm{VD}=4 \mathrm{~mm}$, having $\mathrm{BW}=60 \mathrm{MHz}$. The bandwidth increased by increasing the vertical distance of the third and the fourth conducting levels.

Fig. 6(a) represents the frequency response of an HTS filter with vertical distance $5 \mathrm{~mm}$, showing $\mathrm{BW}=110 \mathrm{MHz}$ and $f_{0}=$ $1.78 \mathrm{GHz}$. The bandwidth was increased to $150 \mathrm{MHz}$ in a tested HTS filter with VD $=6 \mathrm{~mm}$, shown in Fig. 6(b). Table I shows the summary of the tests of this adjustable multilayer HTS filter.
TABLE I

Summary OF THE TeSTS OF AN ADJUSTABLE MUlTILAYER HTS Filter

\begin{tabular}{cccc}
\hline Test & $\begin{array}{c}\text { Center Frequency } \\
f_{0}(\mathrm{GHz})\end{array}$ & $\begin{array}{c}\text { Band Width } \\
\text { BW }(\mathrm{MHz})\end{array}$ & $\begin{array}{c}\text { Insertion Loss } \\
\mathrm{IL}(\mathrm{dB})\end{array}$ \\
\hline 1 & 1.78 & 60 & 0.59 \\
2 & 1.78 & 110 & 0.50 \\
3 & 1.83 & 60 & 0.45 \\
4 & 1.88 & 150 & 0.52 \\
5 & 1.92 & 80 & 0.80 \\
6 & 1.92 & 110 & 0.56 \\
\hline \hline
\end{tabular}

The center of frequency from 1.78 to $1.92 \mathrm{GHz}$ and bandwidth between 60 and $150 \mathrm{MHz}$ were obtained.

Microstrip lines in this multilayer HTS filter were shorted to ground by using Ag-paint at the high-current point. This may decrease the unloaded $Q$ of the packaged HTS filter, as discussed in [6]. Insertion losses of the filters in this experiment may be reduced, if the contact resistance of the ground contacts is decreased.

\section{CONCLUSION}

An adjustable multilayer HTS bandpass filter has been designed and fabricated. Four YBCO thin films deposited on $\mathrm{LaAlO}_{3}$ substrates were stacked and placed into a copper housing to form a packaged multilayer HTS filter. The center of frequency of the filter was varied from 1.78 to $1.92 \mathrm{GHz}$ by moving the HTS films. The bandwidth of the filter was also adjusted by changing the vertical distance of the air gap in a copper housing. The variation of the bandwidth from 60 to $150 \mathrm{MHz}$ was obtained.

\section{REFERENCES}

[1] M. J. Scharen, D. R. Chase, A. M. Ho, A. O'Baid, K. R. Raihn, and R. J. Forse, "Filter subsystem for wireless communications," IEEE Trans. Appl. Superconduct., vol. 7, pp. 3744-3749, June 1997.

[2] G. Roesler, "Superconducting filters enhance military receiver performance," Microwaves RF, p. 61, June 1998.

[3] G. L. Matthaei, N. O. Fenzi, R. Forse, and S. Rohlfing, "Narrow-band hairpin-comb filters for HTS and other applications," in IEEE MTT-S Int. Microwave Symp. Dig., 1996, pp. 457-460.

[4] A. Enokihara and K. Enokihara, " $1.5 \mathrm{GHz}$ high- $T_{c}$ superconducting microstrip bandpass filter of miniaturized configuration," J. Superconduct., vol. 10, p. 49, Feb. 1997.

[5] C. Zuccaro, M. Winter, N. Klein, and K. Urban, "Microwave absorption in single crystal of lanthanum aluminate," J. Appl. Phys., vol. 82, pp. 5695-5704, Dec. 1997.

[6] K. D. Mossman, G. L. Matthaei, and G. L. Hey-Shipton, "A narrow-band HTS bandpass filter at $18.5 \mathrm{MHz}$," in IEEE MTT-S Int. Microwave Symp. Dig., 2000, pp. 653-656.

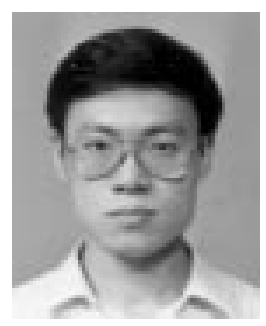

Kouth Chen received the B.S. degree in physics from the National Tsing Hua University, Hsin-Chu, Taiwan, and the M.S. and Ph.D. degrees from Northeastern University, Boston, MA, in 1979, 1984 and 1989 , respectively.

In June 1989, he joined the Materials Research Laboratories, Industrial Technologies Research Institute in Taiwan. His research interests have included high-temperature superconductive material and wire fabrications, magnet and power applications, and superconducting filter in wireless applications. Since November 2000, he has been with the Wang NMR Inc. and the Advanced Superconducting Technologies Co. Ltd. His current interests include the superconductor in medical, power, and wireless applications. 


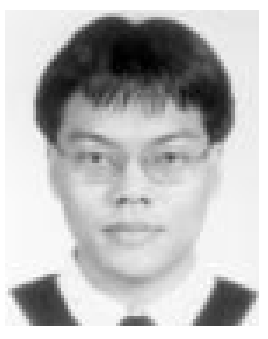

Ming-Jye Chen was born in Taipei, Taiwan, on July 4, 1974. He received the B.S. degree in physics from the National Sun Yat-Sen University, Kaohsiung, Taiwan, and the M.S. degree in fabrication and characterization of high-Tc step edge Josephson junction array and dc SQUID from National Taiwan University, Taipei, Taiwan, in 2000. He is currently pursuing the Ph.D. degree from the Department of Physics, National Taiwan University.

His research interests include the field of superconducting devices and cryoelectronics.

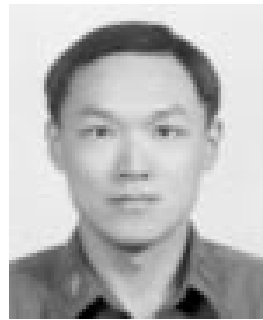

Jau-Han Chen (S'99) was born in Taipei, Taiwan, on February 17, 1971. He received the B.S. degree in physics and the M.S. degree in electrical engineering from National Taiwan University, Taipei, Taiwan, in 1993 and 1995, respectively. Since 1997, he has been pursuing the Ph.D. degree at the university's Department of Physics, where his main research interest is the development of high-Tc superconductor- based devices, especially Josephson devices.

From 1995 to 1997, he served in the Taiwan army. Currently, his research includes the fabrication of high-Tc dc SQUID magnetometers for biomagnetic application and the study of integrated superconducting structures.

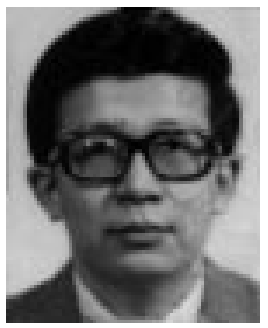

Hong-Chang Yang was born on September 3, 1947 in Chai-Yi, Taiwan. He received the B.S. degree in physics from National Taiwan Normal University, Taipei, Taiwan, the M.S. degree in physics from National Cheng-Kung University, Tainan, Taiwan, and the Ph.D. degree from Iowa State University in 1970, 1974, and 1983, respectively.

From 1974 to 1977, he was an instructor at Tatung University, Taipei, Taiwan. In 1983, he returned to Tatung University as an assistant professor. He later joined the Physics Department at National Taiwan University as an Associate Professor in 1985. He has worked there as a Full Professor since 1989. His research interests include basic research and application of superconductivity, flux dynamics and Josephson effects, SQUIDs, filters, cryoelectronics, thin film technology, and optical property of magnetic fluid thin film. He is the author or coauthor of more than 140 publications in international journals.

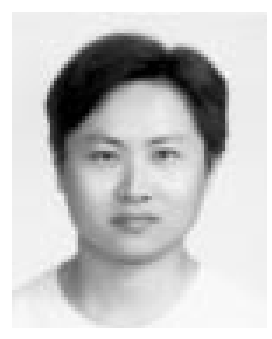

sistance materials.
Li-Min Wang was born in Tainan, Taiwan, on March 26, 1965. He received the B.S. and M.S. degrees in physics from National Taiwan Normal University, Taipei, Taiwan, and the Ph.D. degree from National Taiwan University in 1988, 1990, and 1995, respectively.

From 1995 to 1997, he served in the Taiwan army. Since 1998, he has been an Assistant Professor at Da-Yeh University. His research interests include basic research of superconducting superlattices, Josephson devices, filters, and colossal magnetore-

Chao-Yuan Huang Photograph and biography not available at time of publication.

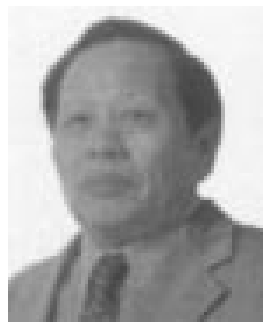

Bert Wang received the B.S. degree in electrical engineering from National Cheng Kung University, Tainan, Taiwan, and the M.S. and Ph.D. degrees in physics from Stanford University, Stanford, CA, in 1963,1967 , and 1970, respectively.

His research interests have included high-energy physics, energy physics, medical physics, and devices. He is a national expert in applied superconductivity, especially in large-scale application, from accelerator technology to fusion, MHD, MRI, NMR, ICR, and functional imagining. He is also a business entrepreneur in applied superconductivity. He founded Wang NMR Inc., U.S. and Advanced Superconducting Technologies Co. Ltd., Taiwan. These two companies engage in production of superconducting devices ranging from medical NMR, MRI, superconducting filter, to power complication in electrical and electronic industries. He has authored about 250 published papers in the above-mentioned area. 